\title{
Fatores associados ao ganho de peso após o ingresso na Universidade
}

Factors associated to weight gain after joining the University

Factores asociados al ganado de peso después del ingreso en la Universidad

\begin{abstract}
Ananda Ivie Dias Novais ${ }^{1}$, Ingrid Bianca Lima de Oliveira ${ }^{2}$, Poliana Cardoso Martins $^{3}$, Verena Macedo Santos ${ }^{4}$, Fernanda Rodrigues de Oliveira Penaforte ${ }^{5}$

\footnotetext{
${ }^{1}$ Nutricionista pela Universidade Federal da Bahia. E-mail: ivie.ananda@gmail.com

2 Nutricionista pela Universidade Federal da Bahia. E-mail: ingrid.blo@hotmail.com

${ }^{3}$ Doutora em Saúde Pública, Docente do Instituto Multidisciplinar em Saúde-Campus Anísio Teixeira da Universidade Federal da Bahia. E-mail: policmartins@yahoo.com.br

${ }^{4}$ Mestre em Ciências Biológicas. E-mail: macedo.verena1@gmail.com

5 Doutor em Investigação Biomédica, Professor adjunto II da Universidade Federal do Triângulo Mineiro. E-mail: fernandapenaforte@yahoo.com.br
}

\section{RESUMO}

O ingresso no ensino superior constitui um momento marcante na vida dos jovens estudantes e representa um período de grandes desafios e mudanças. Estudos evidenciam maior possibilidade de aumento corporal nos primeiros anos da vida universitária levando a alterações no estado nutricional do indivíduo. Este estudo objetivou descrever a prevalência e os fatores associados ao ganho de peso em estudantes de uma instituição federal de ensino superior. Foi realizado um estudo transversal com amostra de 279 estudantes, os dados coletados por meio de questionário semiestruturado e aferidas medidas antropométricas de peso e altura. Para a análise da associação entre as variáveis utilizou-se o modelo de regressão de Poisson com variância robusta. Encontrou-se elevada prevalência de ganho de peso superior a $10 \%$, mudança do perfil nutricional concomitantemente com o aumento do percentual de gordura corpórea, alimentação emocional e a presença da ansiedade como um fator de proteção para esse ganho de peso. Propõe-se estratégias como o mindfulness e o comer intuitivo no auxílio da regulação das emoções e seus reflexos alimentares. Esses achados podem contribuir nas discussões sobre como o ambiente universitário pode influenciar na qualidade de vida do estudante para que esse ambiente se torne mais acolhedor, humano e aberto à discussão.

Palavras-chave: Ganho de peso; Comportamento alimentar; Universidade.

\section{ABSTRACT}

Entering higher education is a defining moment in the lives of young students, representing a period of great challenges and changes. Studies evidenced a greater 


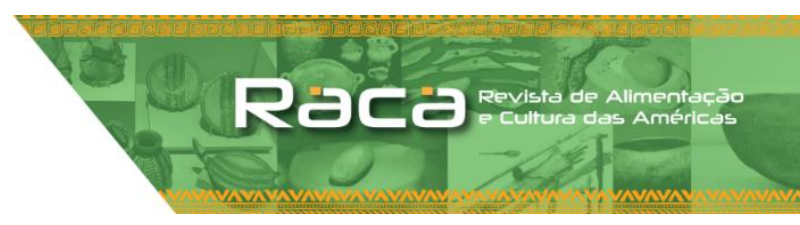

DOI 10.35953/raca.v1i1.17

possibility of corporal increase in the first years of the university life leading to changes in the nutritional state of the individual. This study aimed to describe the prevalence and factors associated with weight gain in students of a federal institution of higher education. A cross - sectional study was carried out with a sample of 279 students, using a semi - structured questionnaire through validated scales. For the analysis of the association between the variables, the Poisson regression model with robust variance was used, and the variables that presented statistical significance of $20 \%$ ( $p<0.20$ ) were selected to be inserted in the multivariate model. For the interpretation of results in the final model, $p \leq 0.05$ was considered. The prevalence of weight gain $>10 \%$ was $29.39 \%$. It was found a high prevalence of weight gain above $10 \%$, change in nutritional profile concomitantly with the increase in body fat percentage, emotional eating and the presence of anxiety as a protective factor for this weight gain. Strategies such as mindfulness and intuitive eating are proposed to help regulate emotions and their eating reflexes. These findings may contribute to discussions about how the university environment can influence student quality of life so that this environment becomes more welcoming, humane and open to discussion.

Keywords: Weight gain; Feeding behavior; Universities.

\section{RESUMEN}

Ingresar a la educación superior es un momento notable en la vida de los jóvenes estudiantes, que representa un período de grandes desafíos y cambios. Los estudios muestran una mayor posibilidad de aumento del cuerpo en los primeros años de vida universitaria que conduce a cambios en el estado nutricional del individuo. Este estudio tuvo como objetivo describir la prevalencia y los factores asociados con el aumento de peso en los estudiantes de una institución federal de educación superior. Se realizó un estudio transversal con una muestra de 279 estudiantes, utilizando un cuestionario semiestructurado a través de escalas validadas. Para el análisis de la asociación entre las variables, se utilizó el modelo de regresión de Poisson con varianza robusta, y las variables con significación estadística del $20 \%$ ( $p$ $<0,20)$ se seleccionaron para ser incluidas en el modelo multivariante. Para la interpretación de los resultados en el modelo final, consideramos $p \leq 0.05$. La prevalencia de aumento de peso $>10 \%$ fue del $29,39 \%$. Se encontró una alta prevalencia de aumento de peso por encima del $10 \%$, cambio en el perfil nutricional concomitantemente con el aumento del porcentaje de grasa corporal, alimentación emocional y la presencia de ansiedad como factor protector para este aumento de peso. Se proponen estrategias como la atención plena y la alimentación intuitiva para ayudar a regular las emociones y sus reflejos alimentarios. Estos hallazgos pueden contribuir a las discusiones sobre cómo el entorno universitario puede influir en la calidad de vida de los estudiantes para que este entorno se vuelva más acogedor, humano y abierto a la discusión.

Palabras clave: Aumento de peso; Comportamiento alimenticio; Universidade 


\section{Introdução}

O ingresso em instituições de ensino superior (IES) constitui um momento de mudanças na vida dos jovens estudantes (1). Dentre elas, destacam-se a redução do nível de atividade física (2), mudança no local de domicílio, renda, responsabilidade pela compra e preparo do alimento, falta de tempo, dentre outros, o que gera mudanças no estilo de vida e no comportamento alimentar do estudante (7), aumentando a possibilidade de ganho de peso, especialmente no primeiro ano de vida universitária ${ }^{(3)}$. Estudos internacionais denominam esse ganho de peso de "freshman 15", fazendo alusão ao ganho ponderal médio de 6,8kg que ocorre nesse período ${ }^{(4)}$, que pode alterar o estado nutricional (EN) do indivíduo, podendo levar ao desenvolvimento de sobrepeso e obesidade ${ }^{(5,6)}$.

Ao ingressarem no ambiente acadêmico, os estudantes também se deparam com uma grande sobrecarga e estresse devido ao aumento da demanda de estudos; a cobrança pessoal, de familiares e professores; e as mudanças decorrentes da transição entre a fase adolescente e adulta, onde, alguns podem desenvolver quadros de ansiedade ${ }^{(8)}$ frente a essas situações. Em alguns casos, esse transtorno reverbera no comportamento alimentar (9) redirecionando suas escolhas alimentares (10).

O comportamento alimentar poderá ser manifestado de maneiras diferentes, podendo ser restritivo (11) (12); descontrolado (13); e/ou emocional (14). Esses comportamentos podem contribuir para o aumento do peso e de gordura corporal, além de estar entre os fatores responsáveis pelo insucesso das tentativas de emagrecimento (12) (13) (15). Além disso, a falta de tempo, de habilidades culinárias e a facilidade do consumo de alimentos industrializados podem corroborar para essas mudanças (49) (50).

Assim, torna-se importante conhecer as modificações ocorridas no EN dos estudantes após seu ingresso no ensino superior no âmbito nacional considerando os potenciais impactos na saúde do ganho de peso. Este estudo se propõe identificar a prevalência de ganho de peso excessivo após o ingresso na universidade e avaliar os fatores de risco associados ao ganho de peso. 


\section{Metodologia}

\section{Delineamento do estudo}

Trata-se de um estudo de caráter descritivo de corte transversal com abordagem quantitativa. Desenvolvido com estudantes dos cursos de graduação de uma instituição federal de ensino superior, no município de Vitória da Conquista-BA.

Para o cálculo amostral foi considerada uma prevalência de $50 \%$, dado a diversidade de eventos, precisão de 5\%, intervalo de confiança de 95\% (IC95\%), efeito de desenho $=1$. No semestre de 2017.2 estavam regularmente matriculados nessa IES 1.068 indivíduos, sendo todos elegíveis para participarem do estudo. Após o cálculo amostral, 283 participaram do estudo. Foram excluídos estudantes (a) com idade inferior a 18 anos, (b) gestantes ou lactantes e (c) aqueles que não participaram das duas etapas do trabalho.

\section{Participantes}

Destaca-se que a seleção dos indivíduos que foram convidados a participar foi realizada através de um sorteio dentro de cada extrato (curso que o estudante estava matriculado). Aqueles que concordaram em participar, assinando o Termo de Consentimento Livre e Esclarecido, foram conduzidos para coleta de dados.

\section{Procedimentos}

Era necessário o autopreenchimento de um questionário semiestruturado que contemplou questões que permitiram a caracterização dos estudantes em características sociodemográficas, estado de saúde, hábitos de vida, comportamento alimentar, que foi avaliado com o auxílio do Three Factor Eating Questionnaire-21 (TFEQR-21) ${ }^{(16)}$ e sinais e sintomas físicos e atuais de ansiedade, com o questionário Inventário de Ansiedade de Beck (BAI) (17). E, uma avaliação antropométrica para caracterização do EN onde foram aferidos peso, altura, circunferências da cintura, quadril e braquial e dobras cutâneas (bíceps, tríceps, 
DOI 10.35953/raca.v1i1.17

supra ilíaca e subescapular). Os valores encontrados foram aplicados em fórmulas e/ou comparados com valores de referência disponíveis na literatura, para classificar o estado nutricional do estudante, através do IMC, classificando inicialmente de acordo a Organização Mundial de Saúde (OMS) e, posteriormente, dicotomizada, e percentual de gordura corpóreos ${ }^{(18)}$.

\section{Avaliações}

Para criação da variável desfecho, foi utilizada a informação sobre o peso atual subtraindo-se o peso que os estudantes referiram ter ao ingressarem e calculou-se $o$ percentual de mudança ponderal. Então, a alteração do peso corporal após o ingresso na universidade foi categorizada em uma mudança significativa quando igual ou superior a 10\% em relação ao peso relatado e não significativa quando tal ganho foi inferior a $10 \%{ }^{(18)}$.

Para definição da cor ou raça foi utilizada a classificação do Instituto Brasileiro de Geografia e Estatística (IBGE), que considera cinco categorias: branca, preta, parda, amarela ou indígena. O nível socioeconômico foi avaliado através do referimento da renda mensal bruta familiar e depois classificado de acordo a quantidade de salários mínimos. A informação sobre os anos de universidade foi obtida de acordo com o ano de ingresso e categorizada em igual ou inferior a dois anos e mais de dois anos.

O nível de ansiedade foi mensurado através do BAI (17), sendo classificado em: 0-10: sintomas mínimos, 11-19: sintomas leves, 20-30: sintomas moderados, 31-63: sintomas graves. E para a escala TFEQR-21, utilizou-se as instruções para construção do score de acordo com Natacci (2011) e estes categorizados em tercis.

Os dados antropométricos foram coletados através da aferição do peso, altura e utilização de pregas cutâneas, utilizando o protocolo de quatro dobras. Para a avaliação do EN, atual e pregresso, foi realizado o Índice de Massa Corporal (IMC) e a Relação Cintura Quadril (RCQ), utilizando a classificação de acordo a Organização Mundial de Saúde (2008), entretanto foram agrupados como dicotômicas. Assim como a variável de percentual de gordura, adaptado de Lohman e, também dicotomizada (19) (21). 


\section{Análise estatística}

Inicialmente, foi feita uma estimativa da prevalência do ganho de peso entre os universitários com intervalo de $95 \%$ de confiança para proporções. Para verificar os fatores associados a este desfecho, realizou-se análise univariada com estimativas de razões de prevalência com cálculo dos respectivos intervalos de confiança. Empregou-se a regressão de Poisson com variância robusta, sendo selecionadas as variáveis que apresentaram significância estatística de 20\% $(p<0,20)$ para serem inseridas no modelo multivariado. $\mathrm{E}$ a identificação de associação estatisticamente significativa para o modelo multivariado de $5 \%(p<0,05)$ entre um determinado fator em estudo e o ganho de peso superior à $10 \%$ do peso corporal foi utilizado para a interpretação dos resultados. A comparação entre modelos foi feita pelo critério de Akaike (AIC). Todas as análises foram realizadas utilizando-se o software Stata versão 12 (Stata Corp., College Station, Estados Unidos) (22).

\section{Comitê de ética}

Em consonância com o disposto na Resolução ํo 466/2012 do Conselho Nacional de Saúde, todos os participantes foram informados sobre os objetivos do trabalho, procedimentos e sigilo dos dados, e manifestaram sua concordância em participar da pesquisa ao assinar o Termo de Consentimento Livre Esclarecido. Esse estudo foi aprovado pelo Comitê de Ética em Pesquisa do Instituto Multidisciplinar em Saúde da Universidade Federal da Bahia (CAEE 81066717.7.0000.5556 05/02/2018).

\section{Resultados}

Ao todo foram entrevistados 283 estudantes, entretanto, a presente análise contou com 279, uma vez que 4 indivíduos se recusaram a fazer as medidas antropométricas. Destes, $82(29,4 \%)$ apresentaram média do ganho de peso superior à $10 \%$ do peso corporal relatado no início do curso. No grupo estudado observa-se um predomínio de mulheres (73,1\%), com idade entre 18 e 24 anos 
DOI 10.35953/raca.v1i1.17

(79,6\%), indivíduos solteiros $(91,0 \%)$, que se auto referiam como parda $(51,1 \%)$ e relataram morar com família ou parentes (58,9\%). 30,2\% dos entrevistados relataram ter renda familiar de até dois salários mínimos e $36,2 \%$ de dois a quatro salários mínimos. Observa-se que $85,6 \%$ relataram estarem satisfeitos/muito satisfeitos com o curso que estão matriculados (Tabela 1).

Tabela 1. Distribuição da população estudada segundo as características do contexto e sociodemográficas. Vitória da Conquista, Brasil, 2018.

\begin{tabular}{|c|c|c|c|}
\hline Variáveis & $\mathrm{n}$ & $\%$ & IC 95\% \\
\hline \multicolumn{4}{|l|}{ Sexo } \\
\hline Masculino & 75 & 26,88 & $21,64-32,11$ \\
\hline Feminino & 204 & 73,12 & $67,88-78,35$ \\
\hline \multicolumn{4}{|l|}{ Idade } \\
\hline $18-24$ & 222 & 79,57 & $74,80-84,33$ \\
\hline $25-30$ & 48 & 17,2 & $12,74-21,66$ \\
\hline 30 ou mais & 9 & 3,23 & $01,13-05,31$ \\
\hline \multicolumn{4}{|l|}{ Renda } \\
\hline Até 2 salários & 71 & 30,21 & $24,29-36,12$ \\
\hline De 2 a 4 salários & 85 & 36,17 & $29,98-42,35$ \\
\hline De 4 a 10 salários & 65 & 27,66 & $21,89-33,42$ \\
\hline Mais de 10 salários & 14 & 5,96 & $02,90-09,00$ \\
\hline \multicolumn{4}{|l|}{ Estado Conjugal } \\
\hline Solteiro & 254 & 91,04 & $87,66-94,41$ \\
\hline Casado/Em união & 23 & 8,24 & $4,99-11,49$ \\
\hline Separado/Divorciado & 2 & 0,72 & $0,28-1,71$ \\
\hline \multicolumn{4}{|l|}{ Raça } \\
\hline Parda & 140 & 51,09 & $45,13-57,05$ \\
\hline Branca & 71 & 25,91 & $20,69-31,13$ \\
\hline Preta & 55 & 20,07 & $15,30-24,84$ \\
\hline Amarela & 6 & 2,19 & $0,44-3,93$ \\
\hline Indígena & 2 & 0,73 & $0,28-1,74$ \\
\hline \multicolumn{4}{|l|}{ Arranjo familiar } \\
\hline $\begin{array}{l}\text { Mora sozinho ou com } \\
\text { amigos }\end{array}$ & 115 & 41,22 & $35,40-47,03$ \\
\hline $\begin{array}{l}\text { Mora com família ou } \\
\text { parentes }\end{array}$ & 164 & 58,78 & $52,96-64,59$ \\
\hline \multicolumn{4}{|l|}{$\begin{array}{l}\text { Satisfação com o } \\
\text { curso }\end{array}$} \\
\hline $\begin{array}{l}\text { Satisfeito/Muito } \\
\text { satisfeito }\end{array}$ & 237 & 85,56 & $81,39-89,72$ \\
\hline $\begin{array}{l}\text { Insatisfeito/Pouco } \\
\text { satisfeito }\end{array}$ & 40 & 14,44 & $10,27-18,60$ \\
\hline \multicolumn{4}{|l|}{ Anos de universidade } \\
\hline Mais de 2 anos & 137 & 49,1 & $43,20-55,00$ \\
\hline Menos de 2 anos & 144 & 50,9 & $44,99-56,79$ \\
\hline
\end{tabular}


DOI 10.35953/raca.v1i1.17

$\begin{array}{lccc}\text { Nutrição } & 39 & 13,98 & 09,88-18,07 \\ \text { Biologia } & 34 & 12,19 & 08,32-16,04 \\ \text { Biotecnologia } & 41 & 14,7 & 10,51-18,87 \\ \text { Farmácia } & 46 & 16,49 & 12,10-20,86 \\ \text { Medicina } & 24 & 8,6 & 05,29-11,91 \\ \text { Enfermagem } & 44 & 15,77 & 11,46-20,07 \\ \text { Psicologia } & 51 & 18,28 & 13,71-22,84\end{array}$

IC: Intervalo de confiança.

Com relação às variáveis de saúde nota-se que 74,4\% da amostra relatou não possuir nenhuma patologia no momento da entrevista, porém apresentaram mudanças no padrão de adoecimento após ingresso na instituição, visto que 57,2\% relatou ficar mais doente atualmente.

Grande parcela consome álcool $(68,2 \%)$, não fuma $(87,8 \%)$ e não utiliza outras drogas $(90,5 \%)$. A prática de dieta já foi realizada por 10,7\% e 22,2\% relataram estar fazendo dietas atualmente.

Segundo a classificação da escala de ansiedade, $28,3 \%$ dos indivíduos apresentaram sintomatologia de ansiedade grave. $E$, no que diz respeito ao comportamento alimentar, apresentaram média de score de 34,14 $\pm 1,27$ para

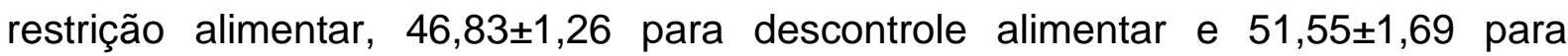
alimentação emocional. Além disso, 34,8\%, 39,07\% e 37,3\%, estão no menor tercil de comportamento restritivo, descontrole alimentar e alimentação emocional, respectivamente (Tabela 2 ). Dentre eles, o maior tercil se apresentou dentro de alimentação emocional (32,6\%).

Tabela 2. Distribuição da população estudada segundo as comorbidades, comportamento alimentar e estilo de vida. Vitória da Conquista, Brasil, 2018.

\begin{tabular}{lccc}
\hline Variáveis & $\mathrm{n}$ & $\%$ & $\mathrm{IC} \mathrm{95 \%}$ \\
\hline $\begin{array}{l}\text { Doença atualmente } \\
\quad \text { Não possui }\end{array}$ & 206 & 74,37 & \\
$\quad \begin{array}{l}\text { Possui } \\
\text { Mudança no padrão de }\end{array}$ & 71 & 25,63 & $20,45-30,80$ \\
$\begin{array}{l}\text { adoecimento pós } \\
\text { ingresso }\end{array}$ & & $69,19-79,54$ \\
$\quad$ Não & 119 & 42,81 & \\
Sim & 159 & 57,19 & $36,95-48,65$ \\
\end{tabular}

Ansiedade 
DOI 10.35953/raca.v1i1.17

Grave
Moderada
Leve
Mínimo
Comportamento
alimentar
Comportamento
Restritivo

Maior tercil

Tercil intermediário

Menor tercil

Descontrole Alimentar

Maior tercil

Tercil intermediário

Menor tercil

Alimentação Emocional

Maior tercil

Tercil intermediário

Menor tercil

\section{Consumo de Álcool}

Sim

Não

Tabagismo

Sim

Não

Droga

Sim

Não

Atividade física

Sim

Não

Prática de dieta

Sim

Não

\section{IMC Atual}

Eutrofia/Magreza

Sobrepeso/Obesidade

IMC pregresso

Eutrofia/Magreza

Sobrepeso/Obesidade

Gordura corporal

Acima da

média/Elevado

Média/Abaixo da média
79

56

88

56

87

88

104

82

88

109

91

91

97

\section{Hábitos de vida}

189

88

33

238

26

248

85

194

30

249
28,32

20,07

31,54

20,07

31,18

31,54

37,28

29,39

31,54

39,07

32,62

32,62

34,77
68,23

87,82

9,49
90,51
22,99-33,63

$15,34-24,80$

26,05-37,02

$15,34-24,80$

25,71-36,65

$26,05-37,03$

$31,56-42,98$

24,01-34,76

26,05-37,02

$33,30-44,82$

27,08-38,15

$27,08-38,15$

$29,14-40,38$

$62,71-73,74$

$26,25-37,28$

08,25-16,09

$83,90-91,74$

$5,99-12,98$

$87,01-94,00$

64,09-74,96

25,03-35,90

07,09-14,41

$85,58-92,90$

\section{Medidas antropométricas}

$\begin{array}{ccc}207 & 74,19 & 69,02-79,35 \\ 72 & 25,81 & 20,64-30,97 \\ 229 & & \\ 50 & 82,08 & 77,55-86,60 \\ & 17,92 & 13,39-22,44 \\ 212 & & \\ 57 & 78,81 & 73,89-83,72 \\ & 21,19 & 16,27-26,10\end{array}$




\section{RCQ}

$\begin{array}{lccc}\text { Com risco associado } & 52 & 18,64 & 14,04-23,23 \\ \text { Sem risco associado } & 230 & 81,36 & 76,76-85,95 \\ \text { Satisfação corporal } & & & \\ \text { Sim } & 200 & 72,73 & 67,43-78,02 \\ \text { Não } & 75 & 27,27 & 21,97-32,56\end{array}$

IC: Intervalo de confiança.

Em relação à avaliação antropométrica observa-se que com relação ao IMC de quando ingressaram 82,1\% está classificada em eutrofia/magreza e 17,9\% em sobrepeso/obesidade. $74,2 \%$ - classifica em eutrofia/magreza, 25,8\% em sobrepeso/obesidade, no que tange ao IMC atual. $78,8 \%$ dos indivíduos apresentaram percentual de gordura corporal acima da média/elevado, porém, sem risco associado de doenças cardiovasculares $(81,4 \%)$ segundo a razão cintura/quadril e, quando questionados sobre a satisfação corporal, relataram não estarem satisfeitos $(72,7 \%)$ (Tabela 2$)$.

A relação entre o IMC de ingresso e o IMC atual, nota-se que 11,8\% dos 229 indivíduos que ingressaram em eutrofia/magreza, hoje apresentam sobrepeso/obesidade, além disso, dos 50 indivíduos que ingressaram na classificação de sobrepeso/obesidade, $90 \%$ ainda permanece nessa faixa, apresentando $p$ valor significativo $(<0,001)$.

$\mathrm{Na}$ análise univariada, as variáveis que foram associadas com o ganho de peso maior ou igual a $10 \%$ foram a satisfação com o curso com razão de prevalência de 1,34 para categoria insatisfeito/pouco satisfeito; menos de dois anos de ingresso com razão de prevalência de 1,23; maior adoecimento após ingresso com razão de prevalência de 0,55; ansiedade, classificada como moderada segundo a BAI, com razão de prevalência de 1,16 considerando o valor de $p \leq 0,200$, (Tabela 4).

Para o comportamento restritivo o tercil intermediário apresentou razão de prevalência de 1,52, o descontrole alimentar de 1,68 para o maior tercil e alimentação emocional com razão de prevalência de 2,39 para o tercil intermediário. A satisfação corporal obteve razão de prevalência de 0,65 para aqueles que não estão satisfeitos quando comparado aos que estão. O IMC atual teve 0,93 vezes maior razão de prevalência para os indivíduos classificados com sobrepeso/obesidade, o percentual de gordura corpórea de 1,38 para o percentual acima da média/mais elevado e a razão cintura-quadril, razão de prevalência de 0,4 
DOI 10.35953/raca.v1i1.17

vezes maior para aqueles com risco associado de eventos cardiovasculares (Tabela 3). A partir dessas variáveis, compomos o modelo multivariado.

Tabela 3. Análise univariada por meio da regressão de Poisson para o ganho de $10 \%$ ou mais do peso corpóreo e fatores associados. Vitória da Conquista, Brasil, 2018.

\begin{tabular}{lcccc}
\hline Variável & $\begin{array}{c}\text { Prevalência de } \\
\text { ganho de }>10 \% \text { do } \\
\text { peso } \\
n(\%)\end{array}$ & RP & IC95\% & P valor \\
Satisfação com o curso & & & & \\
& & & 0,195
\end{tabular}

Satisfação com o curso

Satisfeito/Muito satisfeito $66(27,85)$

Insatisfeito/Pouco

satisfeito

$15(37,50)$

1

Anos de universidade

Mais de 2 anos

$56(40,88)$

Menos de 2 anos

$26(18,31)$

1,34

0,85

Mudança no padrão de adoecimento pós

ingresso

Não

$24(20,17)$

Sim

$58(36,48)$

$\begin{array}{cc}1 & 0, \overline{3} 6- \\ 0,55 & 0,83\end{array}$

\section{Ansiedade}

Grave

$28(35,44)$

$1,10 \quad 0,67$

1

Moderada

$21(37,50)$

$1,16 \quad 0,70$ -

Leve

$15(17,05)$

0,53

1,94

Mínimo

$18(32,14)$

0,29 -

Comportamento

Alimentar

\section{Comportamento \\ Restritivo}

0,96

0

Maior tercil
Tercil intermediário
Menor tercil

$26(28,57)$
$33(36,26)$
$23(23,71)$

1,2

Descontrole Alimentar

$\begin{array}{ccc}23(23,71) & 1 & - \\ 33(40,24) & 1,68 & 1,10- \\ & & 2,58 \\ 23(16,14) & 1,09 & 0,67- \\ 26(23,85) & 1 & 1,78 \\ & & -\end{array}$

Maior tercil

Tercil intermediário

Menor tercil

$26(23,85)$

$1,52 \quad 0,97-$

1,52$$
1
$$$$
0,016
$$

$0,74-$

0,064

$1,10-$

2,58

1,78

0

$$
0,038
$$<smiles></smiles>
a 
DOI 10.35953/raca.v1i1.17

\begin{tabular}{lccc} 
& & & 0,000 \\
$19(18,27)$ & 1,55 & $1,49-$ & \\
$25(28,41)$ & 2,39 & $0,91-$ & \\
$38(43,68)$ & 1 & 2,63 & \\
$65(32,50)$ & 1 & - & 0,085 \\
$16(21,33)$ & 0,65 & $0,40-$ & \\
$49(23,67)$ & 1 & 1,05 & 0,000 \\
$33(45,83)$ & 1,93 & $1,36-$ & \\
$71(33,49)$ & 1,38 & $1,21-$ & 0,011 \\
$8(14,04)$ & 1 & 4,66 & \\
$20(38,46)$ & 1,4 & $0,93-$ & 0,097 \\
$62(27,31)$ & 1 & 2,11 & \\
\hline
\end{tabular}

Maior tercil
Tercil intermediário
Menor tercil
Satisfação corporal
Sim
Não
IMC Atual
Eutrofia/Magreza
Sobrepeso/Obesidade
Gordura corporal
Acima da
média/Elevado
Média/Abaixo da média
RCQ

Alimentação Emocional

Sem risco associado

Com risco associado

RP: Razão de prevalência; IC: Intervalo de confiança.

No modelo final da análise multivariada, considerando o $p \leq 0,05$, se mantiveram associadas ao ganho de peso: o fato de estarem na universidade a mais de 2 anos (RP 2,47), ansiedade no seu nível mais leve (RP 0,43), alimentação emocional com o maior tercil (RP 1,75), IMC atual para sobrepeso/obesidade (RP 1,57) e percentual de gordura acima da média/elevado (RP 1,75) (Tabela 4).

Tabela 4. Análise multivariada por meio da regressão de Poisson para o ganho de $10 \%$ ou mais do peso corpóreo e fatores associados. Vitória da Conquista, Brasil, 2018.

\begin{tabular}{lccc}
\hline Variáveis & RP & IC 95\% & P valor \\
Anos de universidade & 2,47 & $1,63-3,75$ & 0,000 \\
Ansiedade & 0,43 & $0,23-0,79$ & 0,007 \\
Alimentação Emocional & 1,75 & $1,07-2,87$ & 0,024 \\
IMC Atual & 1,57 & $1,09-2,25$ & 0,014 \\
Gordura corporal & 1,75 & $0,93-3,29$ & 0,08 \\
\hline
\end{tabular}

RP: Razão de prevalência; IC: Intervalo de confiança. 


\section{Discussão}

Foram encontrados nesse estudo uma elevada prevalência de ganho de peso superior a $10 \%$, mudança do perfil nutricional concomitantemente com o aumento do percentual de gordura corpórea, alimentação emocional e a presença da ansiedade como um fator de proteção para esse ganho de peso.

A elevada prevalência do ganho de peso superior a $10 \%$ encontrada nesse estudo pode impactar de maneira negativa na vida dessa população, visto que sobrepeso/obesidade atualmente não para de aumentar, juntamente com o comer transtornado. Entretanto, os ideais de magreza pregados pela mídia atual se tornam inalcançáveis e sendo postos como sinônimo de saúde, por isso, este ganho de peso se torna um potencial estigma para desenvolvimento de problemas psicológicos e sociais, para além das consequências clínicas ${ }^{(23)}$.

Dos 229 indivíduos que ingressaram em magreza/eutrofia, 27 desses hoje se encontram na faixa de sobrepeso/obesidade. Além dessa mudança no estado nutricional, houve associação do percentual de gordura corpórea com o ganho de peso superior a $10 \%$ nesses indivíduos indicando uma razão de prevalência 1,75 vezes de apresentarem ganho de peso superior à 10\% dentre aqueles com percentual elevado/acima da média. Estudos apontam que esse ganho de peso nessa fase de transição resultaria em um aumento da massa corporal e medidas antropométricas e, essas medidas seriam acompanhadas de mudanças na ingestão alimentar e manutenção de hábitos sedentários (7) (24) (25).

O termo "freshman 15" foi proposto por Hovell em 1985 como um fenômeno que acometia calouros no seu primeiro ano de ingresso e, estudos complementares a ele apontaram que esse ganho acontecia independentemente de serem vinculados a rede pública ou privada de ensino (26) (27). O mesmo foi observado no nosso estudo, uma IES federal, onde, a razão de prevalência para apresentar o ganho de peso corporal superior à $10 \%$ nos dois primeiros anos de universidade é 2,47 vezes do que entre os estudantes que já estão nos anos finais de graduação (28).

Em seu estudo qualitativo, Greaney (2009) apresentou algumas barreiras relatadas pelos estudantes para o ganho de peso, dentre os eles, a falta de tempo e a falta de acesso imediato a alimentos saudáveis, assim como espaços que 
promovam atividades físicas, favorecem a inatividade física e o aumento de indicadores antropométricos. Também foi apontado que 0 apoio social (principalmente vindo de amigos) é um facilitador para que hábitos de vida, como por exemplo, comer mais frutas ao longo do dia, se tornassem constantes ${ }^{(29)}$.

A estabilidade do peso no início da vida adulta pode ser um fator importante na redução do risco de doença crônica para indivíduos eutróficos e em sobrepeso (30). Ao constatar que o que vêm se estabelecendo dentre a população é o inverso dessa premissa, devemos pensar em estratégias que superem esse ganho e que façam com que os jovens incorporem práticas saudáveis de vida e, quanto mais cedo houver essa adoção de condutas positivas em saúde, maiores as chances desses hábitos persistirem ao longo de toda sua vida o que irá gerar benefícios para a saúde pública em geral (31).

Para além, deve-se pensar que o ganho de peso pode influenciar negativamente a inserção do indivíduo na sociedade devido as novas concepções e crenças culturais com relação ao corpo magro ${ }^{(32)}$ e corrobora para um comer transtornado com a comida, o que pode gerar distúrbios severos no comportamento alimentar do indivíduo (33).

No que tange ao comportamento alimentar, observou-se a razão de prevalência 0,75 vezes maior de indivíduos que apresentam alimentação emocional ganhar mais de $10 \%$ do peso corpóreo. O comportamento alimentar vem sendo descrito como atitudes relacionadas às práticas alimentares associadas a aspectos socioculturais que estejam envolvidas com o ato de se alimentar ou com o alimento em si, como características intrínsecas ao indivíduo ${ }^{(34)}$.

O comportamento restritivo consiste em uma posição mental adotada em relação aos alimentos na tentativa de reduzir sua ingestão energética (11) (12); o descontrolado, caracterizado pela perda do autocontrole e consumo exagerado de alimentos, com ou sem a presença de fome ${ }^{(13)}$; e/ou emocional, onde o indivíduo irá buscar obter conforto através do alimento ${ }^{(14)}$, principalmente nos alimentos de alta patabilidade. Esses padrões no comportamento alimentar disfuncional tendem a surgir com frequência como válvula de escape para frustrações do rendimento acadêmico e, também como compensação para as exigências impostas ${ }^{(35)}$. 
Alguns estudos não mostram evidências de variação de medidas antropométricas na presença da alimentação emocional, sugerindo que tal padrão de comportamento alimentar pode ter efeito semelhante sobre indivíduos dentro ou acima do peso (36) (37). Além disso, cursar graduação em áreas da saúde não foi indicativo de alimentação mais "saudável" (51). Em contrapartida, Garaulet (2012), encontrou maior influência emocional no comportamento alimentar dentro de grupo de obesos, propondo que a forma como a emoção é sentida está diretamente ligado a hiperfagia, favorecendo na maioria das vezes o consumo de alimentos doces e ricos em gorduras ${ }^{(38)}(40)$.

Essa perturbação do comportamento alimentar, associada a sentimentos negativos como frustração, depressão e ansiedade, podem desencadear outros problemas alimentares como a compulsão alimentar que inclui o ato de comer muito mais rápido do que o normal, comer grandes quantidades de comida na ausência da fome e logo após se sentir constrangido, culpado e triste (41) (43).

Dessa maneira, estudos vêm propondo o uso de mindfulness, podendo ser definida como a capacidade intencional de trazer atenção ao momento presente, sem julgamentos ou críticas. Nesse sentido, o desenvolvimento do comer com atenção plena é de suma importância como estratégia da regulação da emoção do indivíduo e do reflexo da ingestão alimentar. Assim como estratégias do comer intuitivo para que aprendam a confiar em sua habilidade de diferenciação de suas sensações emocionais e físicas para adquirir sabedoria corporal afim de promover mudança no comportamento alimentar (23) (44) (45).

A ansiedade, sintomatologia leve, de acordo com o inventário de ansiedade de Beck ${ }^{(46)}$, foi associada como um fator de proteção para o ganho de mais de $10 \%$ do peso corpóreo. Não foi encontrado nenhum estudo na literatura que corroborasse com esse achado, sendo uma variável passível de maior exploração e investigação dos fatores de confundimento possíveis. O que se pode predizer é que devido à sintomatologia de ansiedade, como taquicardia, tontura, dor de cabeça, formigamento, sudorese, tensão e angústia, o indivíduo passe a perceber seu corpo e tentar ter um maior controle sobre sua saúde.

Vem sendo apontado na literatura que os estudantes de curso da área da saúde apresentam maiores níveis de sintomatologia de ansiedade quando 
DOI 10.35953/raca.v1i1.17

comparadas a outras áreas de ensino, porém, a presença de níveis de ansiedade leves e moderados ajudam no processo de aprendizagem, no raciocínio, na percepção e aumentam a concentração (47) (48).

Destaca-se o fato de ser um estudo longitudinal, o que impossibilita a relação de causa-efeito e também não abrange a incidência, não determinando o risco absoluto e a duração do desfecho, dessa forma outros estudos devem ser desenvolvidos a fim de acompanhar as possíveis alterações que ocorrem no ganho de peso em jovens adultos após o ingresso na universidade. Outra limitação diz respeito a forma de mensuração de algumas variáveis com relação a outros estudos publicados devido a utilização de diferentes métodos e técnicas para investigação do ganho de peso.

\section{Considerações finais}

Os resultados obtidos nessa pesquisa apontam elevada presença de disfunções na sintomatologia da ansiedade, comportamento alimentar, mudança no padrão do IMC, elevado percentual de gordura corporal e alta insatisfação corpórea. Além disso, o ganho de peso de $10 \%$ ou mais de peso corpóreo se apresentou associado ao tempo de ingresso na universidade. O estigma relacionado ao ganho de peso pode aumentar a insatisfação corporal e não predispõe maior motivação para perda do peso. Esses dados são preocupantes pois trata-se de estudantes universitários de um Instituto Multidisciplinar em Saúde, onde, se prega a adoção de hábitos saudáveis para uma melhor qualidade e consequente expectativa de vida.

Desse modo, o uso de estratégias como o mindfulness e o comer intuitivo podem auxiliar na regulação das emoções e seus reflexos alimentares. Para além disso, esses achados podem contribuir nas discussões sobre como o ambiente universitário pode influenciar na qualidade de vida do estudante para que esse ambiente se torne mais acolhedor, humano e aberto à discussão já que o ganho de peso nessa fase da vida pode reverberar por muitos anos de maneira negativa. 


\section{Referências}

(1) Almeida LS, Soares AP, Paisana J. Rendimento acadêmico no ensino superior: estudo com alunos do $1^{\circ}$ ano. 2007;14.

(2) Butler SM, Black DR, Blue CL, Gretebeck RJ. Change in diet, physical activity, and body weight in female college freshman. Am J Health Behav. 2004;28(1):24-32.

(3) Fedewa M V, Das BM, Evans EM Dishman RK. Change in Weight and Adiposity in College Students. Am J Prev Med [Internet]. Elsevier; 2014;47(5):64152. Recuperado de: http://dx.doi.org/10.1016/j.amepre.2014.07.035

(4) Morrow ML, Heesch KC, Dinger MK, Hull HR, Kneehans AW, Fields DA. Freshman 15: Fact or fiction? Obesity. 2006;14(8):1438-43.

(5) Cluskey M, Grobe D. College weight gain and behavior transitions: male and female differences. J Am Diet Assoc [Internet]. American Dietetic Association; 2009;109(2):325-9. Recuperado de: http://dx.doi.org/10.1016/j.jada.2008.10.045

(6) Racette SB, Deusinger SS, Strube MJ, Highstein GR, Deusinger RH. Weight Changes, Exercise, and Dietary Patterns During Freshman and Sophomore Years of College. J Am Coll Heal. 2010;53(6):245-51.

(7) Poddar KH, Hosig KW, Nickols-Richardson SM, Anderson ES, Herbert WG, Duncan SE. Low-Fat Dairy Intake and Body Weight and Composition Changes in College Students. J Am Diet Assoc [Internet]. American Dietetic Association; 2009;109(8):1433-8. Recuperado de: http://dx.doi.org/10.1016/j.jada.2009.05.005

(8) Santos RM. Perfil de ansiedade em estudantes universitários de cursos da área da saúde. 2014;86. Recuperado de:

http://tede.bc.uepb.edu.br/jspui/bitstream/tede/2269/2/PDF - Rômulo Moreira dos Santos.pdf

(9) Vieira VCR, Priore SE, Ribeiro SMR, Franceschini SDCC, Almeida LP. Perfil socioeconomico, nutricional e de saude de adolescentes recem-ingressos em uma universidade publica Brasileira. Rev Nutr. 2002;15(3):273-82.

(10) Oliver G, Wardle J. Perceived effects of stress on food choice. Physiol Behav. 1999;66(3):511-5.

(11) Lowe MR, Timko CA. What a difference a diet makes: Towards an understanding of differences between restrained dieters and restrained nondieters. Eat Behav. 2004;5(3):199-208.

(12) Herman CP Mack D. Restrained and unrestrained eating. J Pers. 1975;43(4):647-60.

(13) Gilhooly CH, Das SK, Golden JK, McCrory MA, Dallal GE, Saltzman E, et al. Food cravings and energy regulation: The characteristics of craved foods and their relationship with eating behaviors and weight change during 6 months of dietary energy restriction. Int J Obes. 2007;31(12):1849-58.

(14) Rutters F, Nieuwenhuizen AG, Lemmens SGT, Born JM, Westerterpplantenga MS. Behavior and Psychology. 2009;17(1):1-6. 
(15) Perrot $M$. La restriction cognitive ou comment les régimes font grossir Description de la situation. Concepts Outil. 2016;128-34.

(16) Natacci LC, Ferreira Júnior $M$. The three factor eating questionnaire - R21: tradução para o português e aplicação The three factor eating questionnaire - R21: translation and administration to Brazilian women. Rev Nutr. 2011;24(3):383-94.

(17) Cunha JA. Manual da versão em português das escalas Beck. São Paulo: Casa do Psicólogo; 2001.

(18) Blackburn LG, Bistrian RB, Maini SB, Smith FM, Schalamm TH. Nutritional and metabolic assessment of the hospitalized patient. Parenter Enter Nutr. 1977;

(19) Pires R, Oliveira de SJ, Torres T, Siqueira da SCE, Belem M. Perfil antropométrico e de composição corporal de lutadores de combate. Importância do diagnóstico correto. Rev Digit EFDeportes.com. 2011;16:156.

(20) WORLD HEALTH ORGANIZATION. Obesity: preventing and managing the global epidemic. Report of a WHO Consultation. Geneva; 2008.

(21) Lohman TG. Advances in Body Composition Assessment. Hum Kinet Publ. 1992;

(22) College Station. Stata Corp. Estados Unidos;

(23) Alvarenga M, Koritar P. Atitude e comportamento alimentar - determinantes de escolhas e consumo. Nutrição Comportamental. 2015. 1261 p.

(24) Pullman AW, Masters RC, Zalot LC, Carde LE, Saraiva MM, Dam YY, et al. Effect of the transition from high school to university on anthropometric and lifestyle variables in males Presented in part at the Canadian Society for Nutritional Sciences, Canadian Nutrition Congress, held in Winnipeg, Manitoba, from 18-21 June 2007. Appl Physiol Nutr Metab [Internet]. 2009;34(2):162-71. Recuperado de: http://www.nrcresearchpress.com/doi/abs/10.1139/H09-007

(25) Hodge CN, Jackson LA, Sullivan LA. The "Freshman 15 " Facts and Fantasies About Weight Gain in Co//ege Women. 1993;17:119-26.

(26) Hoffman DJ, Policastro P, Quick V, Lee S-K. Changes in Body Weight and Fat Mass of Men and Women in the First Year of College: A Study of the "Freshman 15". $J$ Am Coll Heal [Internet]. 2006;55(1):41-6. Recuperado de: http://www.tandfonline.com/doi/abs/10.3200/JACH.55.1.41-46

(27) Lloyd-Richardson EE, Bailey S, Fava JL, Wing R. A prospective study of weight gain during the college freshman and sophomore years. Prev Med (Baltim) [Internet]. Elsevier Inc.; 2009;48(3):256-61. Recuperado de: http://dx.doi.org/10.1016/j.ypmed.2008.12.009

(28) Hovell MF, Mewborn CR, Randle Y, Fowler-Johnson S. Risk of excess weight gain in university women: A three-year community-controlled analysis. Addict Behav. 1985;10(1):15-28.

(29) Greaney ML, Less FD, White AA, Dayton SF, Riebe D, Blissmer B, et al. College Students' Barriers and Enablers for Healthful Weight Management: A Qualitative Study. J Nutr Educ Behav [Internet]. Elsevier Inc.; 2009;41(4):281-6. Recuperado de: http://dx.doi.org/10.1016/j.jneb.2008.04.354 


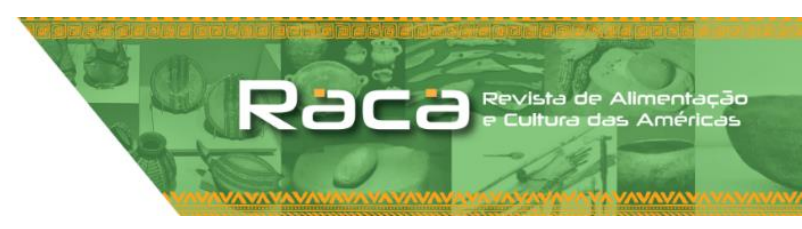

DOI 10.35953/raca.v1i1.17

(30) Strong K a, Parks SL, Anderson E, Winett R, Davy BM. Weight Gain Prevention: Identifying Theory-Based Targets for Health Behavior Change in Young Adults. J Am Diet Assoc. 2008;108(10):1708-15.

(31) França C da, Colares V. Validação do National College Health Risk Behavior Survey para utilização com universitários brasileiros. Cien Saude Colet [Internet]. 2010;15 Suppl 1(supl.1):1209-15. Recuperado de:

http://www.scielo.br/pdf/csc/v15s1/030.pdf\%0Ahttp://www.scielo.br/scielo.php?script= sci_arttext\&amp\%5Cnpid=S1413-81232010000700030

(32) Amparo L, Santos S. O corpo, o comer e a comida. 2008. 330 p.

(33) Vilhena J De, Novaes JDV. Comendo, comendo e não se satisfazendo: apenas uma questão cirúrgica? Obesidade mórbida e o culto ao corpo na sociedade contemporânea. Rev Mal-Estar e Subjetividade [Internet]. 2008;VIII(2):379-406.

Recuperado de: http://pepsic.bvsalud.org/scielo.php?script=sci_arttext\&pid=S151861482008000200006

(34) Vaz DSS, Bennemann RM. Comportamento Alimentar E Hábito Alimentar: Uma Revisão Eating Behavior and Food Habit: a Review. Rev UNINGÁ Rev [Internet]. 2014;20(1):108-12. Recuperado de:

http://www.mastereditora.com.br/review

(35) Marques SIP. Perturbação de Ingestão Compulsiva , Alimentação Emocional e Síndrome do Comer Noturno Um estudo comparativo entre Sujeitos com Peso Normal , Excesso de Peso e Obesidade Psicologia Clínica e da Saúde. 2013;

(36) Kenardy J, Butler A, Carter C, Moor S. Eating, mood, and gender in a noneating disorder population. Eat Behav. 2003;4(2):149-58.

(37) O'Connor NE, Grabowski JH, Ladwig LM, Bruno JF. Simulated predator extinctions: Predator identity affects survival and recruitment of oysters. Ecology. 2008;89(2):428-38.

(38) Konttinen H, Silventoinen K, Sarlio-Lahtenenkorva S, Mannistö S, Haukkala A. Emotional eating and physical activity self-efficacy as pathways in the association between depressive symptoms and adiposity indicators $1-3$. Am $\mathrm{J}$ Clin Nutr. 2010;92(5):1031-9.

(39) Macht M. How emotions affect eating: A five-way model. Appetite. 2008;50(1):1-11.

(40) Garaulet M, Canteras M, Morales E, López-Guimera G, Sánchez-Carracedo D, Corbalán-Tutau MD. Validación de un cuestionario de comedores emocionales para uso en casos de obesidad; cuestionario de comedor emocional (CCE). Nutr Hosp. 2012;27(2):645-51.

(41) American Psychiatric Association. Feeding and Eating Disorders. 2014;1-26.

(42) de Zwaan M. Binge eating disorder and obesity. Int J Obes Relat Metab Disord [Internet]. 2001;25 Suppl 1:S51-5. Recuperado de: https://www.ncbi.nlm.nih.gov/pubmed/11466589

(43) De Man Lapidoth J, Ghaderi A, Norring C. Binge eating in surgical weight-loss treatments. Long-term associations with weight loss, health related quality of life 
(HRQL), and psychopathology. Eat Weight Disord. 2011;16(4):263-9.

(44) Quinto da Luz F, da Silva Oliveira M. Terapia cognitivo-comportamental da obesidade: uma revisão da literatura Cognitive-behavioral therapy of obesity: a literature review. Aleth 40 [Internet]. 2013;jan/abr(2009):159-73. Recuperado de: http://pepsic.bvsalud.org/pdf/aletheia/n40/n40a14.pdf

(45) Bays JC. Mindful Eating: A Guide to Rediscovering a Healthy and Joyful Relationship with Food. Shambhala Publications; 2016. 175 p.

(46) Weingarten HP, Elston D. Food cravings in a college population. Appetite. 1991;17(3):167-75.

(47) Dehghan-nayeri N, Adib-Hajbaghery M. Effects of progressive relaxation on anxiety and quality of life in female students: A non-randomized controlled trial. Complement Ther Med [Internet]. Elsevier Ltd; 2011;19(4):194-200. Recuperado de: http://dx.doi.org/10.1016/j.ctim.2011.06.002

(48) Ribeiro BS, Luiz R, Rodrigues DA, Fernando S, Duarte P. Artigo Prevalência e Fatores Associados com o Consumo de Benzodiazepínicos por Acadêmicos de Enfermagem e Farmácia de uma Faculdade Particular do Sudoeste da Bahia Prevalence and Factors Associated with the Consumption of Benzodiazepínes by Academics of N. Rev Multidiscip e Psicol. 2017;166-76.

(49) Alves Hayda Josiane, Boog Maria Cristina Faber. Comportamento alimentar em moradia estudantil: um espaço para promoção da saúde. Rev. Saúde Pública [Internet]. 2007 Apr [cited 2019 Feb 10] ; 41( 2 ): 197-204. Available from: http://www.scielo.br/scielo.php?script=sci_arttext\&pid=S003489102007000200005\&lng=en. http://dx.doi.org/10.1590/S003489102007000200005.

(50) Feitosa EPS, Dantas CAO, Andrade-Wartha ERS, Marcellini PS, MendesNetto RS. Hábitos alimentares de estudantes de uma universidade pública no Nordeste, Brasil. Rev Alim Nutr 2010; 21(2):225-230.

(51) BERNARDO Greyce Luci, JOMORI Manuela Mika, FERNANDES Ana Carolina, PROENÇA Rossana Pacheco da Costa. Food intake of university students. Rev. Nutr. [Internet]. 2017 Dec [cited 2019 Feb 10] ; 30 ( 6 ): 847-865. Available from: http://www.scielo.br/scielo.php?script=sci_arttext\&pid=S141552732017000600847\&lng=en. http://dx.doi.org/10.1590/1678-98652017000600016. 